\title{
Mastering the Multi-scale Challenge: A Modern Correlation Environment
}

\author{
Arno P. Merkle ${ }^{1}$, Lorenz Lechner ${ }^{1}$, Andy Steinbach ${ }^{1}$, Jeff Gelb ${ }^{1}$, Fabian Perez-Willard ${ }^{2}$, \\ Michael W. Phaneuf ${ }^{3}$, David Unrau ${ }^{3}$ \\ ${ }^{1}$ Carl Zeiss X-ray Microscopy, Inc. 4385 Hopyard Road, Pleasanton, CA, USA \\ ${ }^{2}$ Carl Zeiss Microscopy, GmbH, Oberkochen, Germany \\ ${ }^{3}$ Fibics Incorporated, Ottawa, Ontario, Canada
}

In most fields of study, it is imperative to understand the behavior of a system across several length scales in three dimensions in order to properly address the structural parameters that govern its performance. In order to characterize a system, be it the brain, a structural metal alloy, or a porous rock reservoir, multiple microscopic methods have evolved to specialize in capturing a relatively well defined window of scales, modalities or dimensions of information. Examples of this include medical-CT, confocal light microscopy, X-ray microscopy (XRM), FIB-SEM tomography, serial block face SEM, TEM tomography, atom probe tomography, and more. As these techniques have progressed individually, a clear challenge that has emerged has been how to intelligently and efficiently navigate to and acquire 3D volumes of interest (from centimeter to nanometer), and, subsequently, to fuse multi-scale and multimodality datasets in such a way that leaves the microscopist in control, as recently published in the context of a corrosion study [1].

Here we present the emergence of a major development enabling efficient correlation across modalities, length scales, and dimensions. ZEISS Atlas 5, released in 2015, is a modern, advanced SEM and FIB-SEM acquisition system combined with a modern correlative microscopy workflow environment which acts as the common hub and interface between experiments performed across multiple platforms (SEM, LM, FIB-SEM, XRM, etc.). Atlas 5 automates several advanced SEM and FIB-SEM acquisition tasks, but also provides a visualization environment to co-locate, calibrate and register multiple datasets from multiple instruments in one place. Going further, this environment extends beyond the conventional 2D correlation approach, by incorporating 3D datasets such as those obtained by XRM or FIB-SEM tomography.

We review workflows that are enabled in the context of three examples, from Materials Research, Life Science and Geoscience. In Materials Science, XRM tomograms collected on an Al 7075 aluminum alloy (Figure 1) are used to locate inclusions and pores within the interior of the microstructure, which are then selected and examined at higher resolution by targeted FIB-SEM serial sectioning at defined regions of interest [4]. In Life Sciences, XRM presents a unique opportunity to bridge the length scales between light and electron microscopy (Figure 2), easing the 'needle in a haystack' navigation problem for locating the same region of interest using multiple microscopes [5]. In Geosciences, we demonstrate the great 2D and 3D multi-scale challenge, and how Atlas 5 has been successfully used to enable the efficient study of such porous media systems. 
References:

[1] Burnett T., et. al. Correlative Tomography, Scientific Reports, 2014

[2] A. P. Merkle and J. Gelb, Ascent of 3D X-ray Microscopy in the Laboratory, Microscopy Today, 21 (2013), p. 10

[3] E Maire and P Withers, Quantitative X-ray tomography, International Materials Review, 59 (2014), p. 1

[4] A. P. Merkle et al., Automated Correlative Tomography Using XRM and FIB-SEM to Span Length Scales and Modalities in 3D Materials, Microscopy and Analysis, 28 (2014), p. S10-S13

[5] E. Bushong et al., X-Ray Microscopy as an Approach to Increasing Accuracy and Efficiency of Serial Block-Face Imaging for Correlated Light and Electron Microscopy of Biological Specimens, Microscopy and Microanalysis (2014).

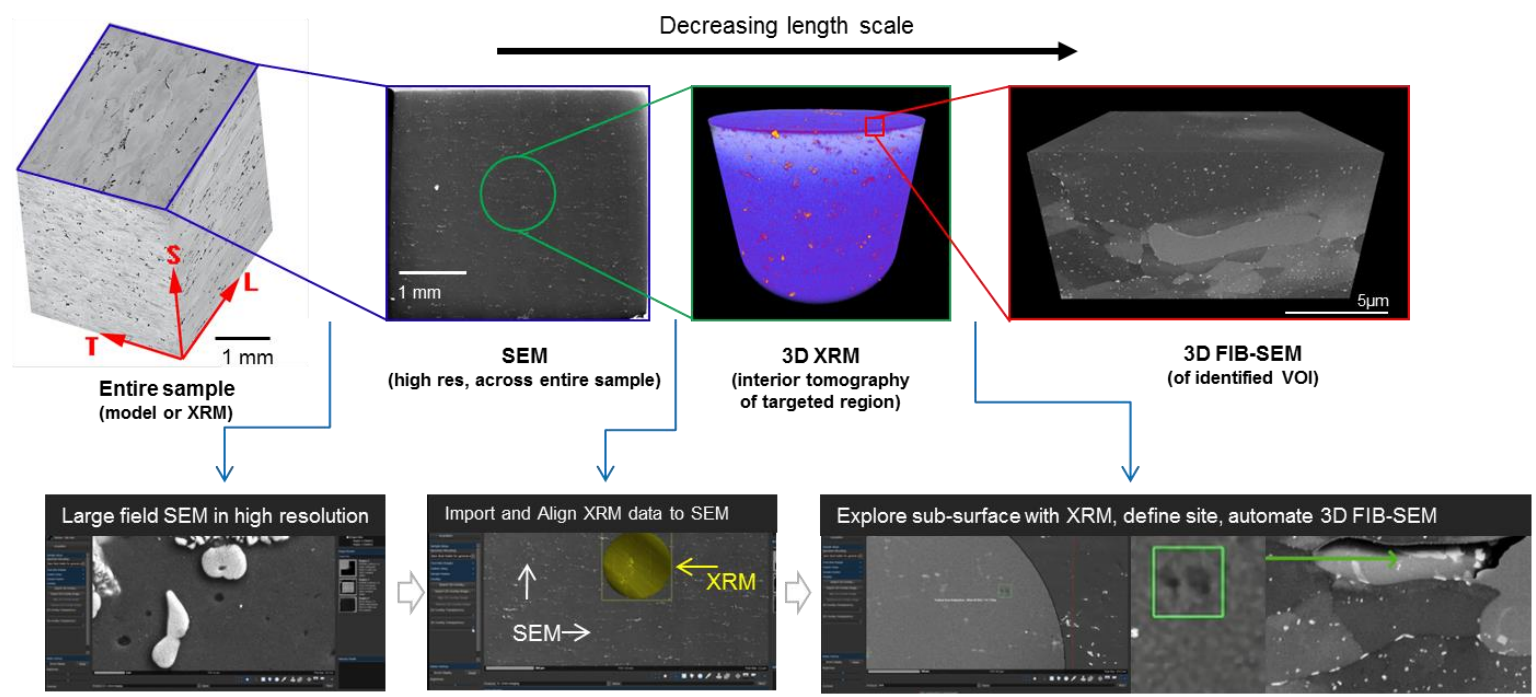

Figure 1: A 3D automated correlative workflow demonstrated on an Aluminum 7075 alloy to access information about inclusions, voids, precipitates and the Al matrix grain structure. In collaboration with N. Chawla and S. Singh of Arizona State University.

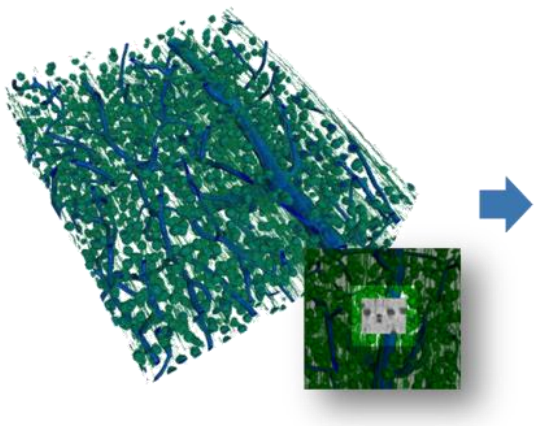

XRM (tomography) Locate Cell Doublet (inset)

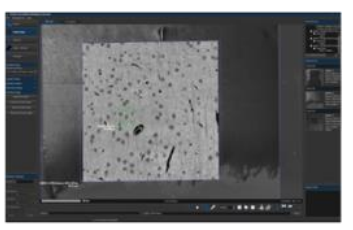

ATLAS

Register, Navigate and Drive Crossbeam to Cell Doublet

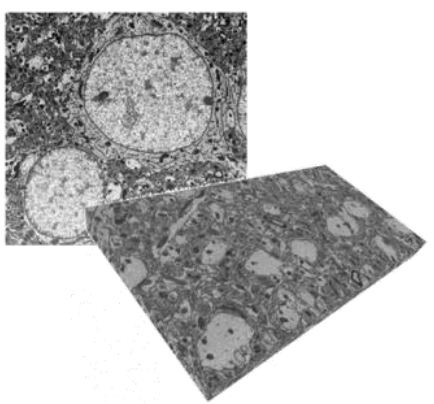

FIB-SEM (tomography) Efficiently located cell doublet

Figure 2: XRM dataset of stained (for EM) mammalian brain tissue, used to navigate to specific subsurface volumes of interest quickly, thereby multiplying the efficiency of 3D EM techniques. In collaboration with NCMIR at the University of California San Diego. 